\title{
Recovery of failed solid-state anaerobic digesters
}

Liangcheng Yang ${ }^{1}$, Xumeng $\mathrm{Ge}^{2}$, Yebo $\mathrm{Li}^{2 *}$

${ }^{1}$ Department of Health Sciences, Illinois State University, 324 Felmley Hall, Normal, IL 61790, USA. Phone: +1 309438 7133. E-mail lyang@ilstu.edu

${ }^{2}$ Department of Food, Agricultural and Biological Engineering, The Ohio State University/Ohio Agricultural Research and Development Center, 1680 Madison Ave., Wooster, OH 44691-4096, USA

* Corresponding author. Phone: +1 330263 3855. E-mail li.851@ osu.edu

\section{Abstract}

This study examined the performance of three methods for recovering failed solid-state anaerobic digesters. The 9-L digesters, which were fed with corn stover, failed at a feedstock/inoculum (F/I) ratio of 10 with negligible methane yields. To recover the system, inoculum was added to bring the F/I ratio to 4 . Inoculum was either added to the top of a failed digester, injected into it, or well-mixed with the existing feedstock. Digesters using top-addition and injection methods quickly resumed and achieved peak yields in 10 days, while digesters using well-mixed method recovered slowly but showed 50\% higher peak yields. Overall, these methods recovered $30-40 \%$ methane from failed digesters. The well-mixed method showed the highest methane yield, followed by the injection and top-addition methods. Recovered digesters outperformed digesters had a constant F/I ratio of 4 . Slow mass transfer and slow growth of microbes were believed to be the major limiting factors for recovery.

Keywords: digester failure, recovery, mass transfer, solid-state anaerobic digestion 


\section{Introduction}

48 There has been growing interest in developing solid-state anaerobic digestion (SS-AD), which can be used with solid contents greater than $15 \%$, due to its high organic loading rate, small digester volume, and high volumetric methane production (Li et al., 2011). A recent study showed that for new digestion projects in Europe, SS-AD has been preferred over conventional low solid anaerobic digestion (Karthikeyan \& Visvanathan, 2013). A major challenge of SS-AD is low mass transfer efficiency between inoculum and feedstock, due to the high solids content, which leads to a series of problems such as accumulation of inhibitors, imbalance of nutrients, and low methane yield (Fagbohungbe et al., 2015; Yang et al., 2014). It was found that the mass diffusion rate of digesting material decreased by 3.7 times when the solid content increased from $8 \%$ to $25 \%$ (Bollon et al., 2011). The high solid content of 30-35\% may also hinder gas-liquid transfer, thus leading to accumulation of carbon dioxide and hydrogen that may inhibit microbes. These potential problems contribute to instability in SS-AD systems, which may eventually cause digester failure (Graef \& Andrews, 1974). and so far there is no single effective solution to prevent failure. Li et al. (2014) tested a variety 
warning for digestion failure. Also, due to high solid content, feedstock in failed digesters cannot

71 be pumped out, but has to be removed by using toe tip buckets, which is a slow and expensive

72 process. Given the possibility and economic cost of digester failure, an efficient and cost-

73 effective recovery plan is needed, especially for large scale solid-state anaerobic digesters.

Recovery within the reactor of a failed digester would be the preferred solution. Theoretically, adding a large amount of inoculum into a failed digester should resume methane production. Inoculum addition has been suggested as method to provide active digestion microbes and dilute inhibitors (e.g. volatile fatty acids), therefore, helps recovering failed digesters (Aboudi et al., 2016). In a previous study, directly adding extra inoculum to the top of failed digesters without

80 further mixing resumed biogas production (Zhu et al., 2014). Besides inoculum, other agents

81 such as alkaline has been applied (Liew et al., 2011). For low solid continuous digesters,

82 recovery of failed digesters may be accomplished by ceasing feeding or re-inoculation with a well-adapted inoculum (Aboudi et al., 2016). The exchange of digesting sludge between serial reactors has also been demonstrated as an effective approach for transferring excess volatile fatty

85 acids from the first reactor to other reactors, thereby recovering the failed digester (Kafle \& Kim, 86 2011). Two concerns for recovery of solid-state anaerobic digesters are the availability of space within 89 the digester and the energy required for mixing. The available space determines the maximum 90 amount of inoculum or other recovery agents that can be added into a failed digester; while the 91 method for mixing the recovery agent with existing digestion feedstock can affect energy 92 demand and recovery outcomes. Previous modeling studies suggested that uniform inoculum 
93 distribution via complete mixing would outperform non-uniform inoculum distribution (Vavilin

94 et al., 2008). However, well-mixing of the recovery agent with the existing feedstock can be a

95 practical challenge due to high solids content and high viscosity. Thus for the best results, the

96 recovery agent and feedstock need to be mixed outside of the digester. Well-mixing also requires

97 high energy inputs.

98

99 In this study, the effects of three methods of adding inoculum to recover a failed digester were

100 tested: top-addition, injection, and well-mixing. Top-addition refers to adding recovery agent (in

101 this test, was inoculum) directly onto the top of the feedstock in the failed digester, injection

102 refers to injecting the inoculum into the central area of the failed digester, and well-mixing refers

103 to thoroughly mixing the added inoculum with the feedstock. A limited amount of inoculum was

104 used for recovery to avoid space concerns. The objectives of this study were to: (1) examine the

105 performance of the three recovery methods in recovering failed solid-state anaerobic digesters,

106 and (2) explore limiting factors during recovery.

\section{2. Methods and materials}

$109 \quad 2.1$ Feedstock and inoculum

110 Corn stover collected from a research farm at the Ohio Agricultural Research and Development

111 Center (OARDC) in Wooster, OH, USA, in January 2013 was used as the feedstock in this study.

112 Corn stover was air dried to less than $15 \%$ moisture content, and then ground to pass a $6 \mathrm{~mm}$

113 sieve (Mighty Mac, MacKissic Inc., Parker Ford, PA, USA). Effluent taken from a mesophilic

114 liquid anaerobic digester (fed with municipal sewage sludge and food wastes, operated by quasar

115 energy group in Zanesville, OH, USA) was used as the inoculum. The inoculum was activated in 
116 a $37^{\circ} \mathrm{C}$ incubation room for one week prior to use. Characteristics of the feedstock and inoculum 117 are shown in Table 1.

1192.2 Anaerobic digestion

120 The SS-AD recovery test was carried out in 9-L plastic digesters (ID: $15 \mathrm{~cm}$, Height: $52 \mathrm{~cm}$, Fig. 121 1) without leachate or biogas recirculation in a $37^{\circ} \mathrm{C}$ incubation room. The corn stover $(400 \mathrm{~g})$ 122 was mixed with inoculum and then loaded into the digesters, with an effective height of about 30 $123 \mathrm{~cm}$ (effective volume of 5.3 L). The mixing ratio was based on the volatile solid content of the 124 feedstock and inoculum. The initial F/I ratio (feedstock/inoculum) was 10, and the initial total 125 solids (TS) content was 32\%. With the high F/I ratio and TS, digesters would most likely fail due 126 to insufficient inoculum supply and retarded mass transfer (Sheets et al., 2015; Zhu et al., 2014). 127 Recovery of failed digesters was carried out on day 20, by adding inoculum into the digester to 128 lower the F/I ratio to 4 and decrease TS to 20 (Table 2). As shown in Figure 1, the inoculum was 129 added in one of three ways: (1) Inoculum was added directly onto the top of the feedstock in the 130 digester (top-addition), so that the inoculum would gradually infiltrate the feedstock. (2) A 131 plastic rod was used to create a $2.54 \mathrm{~cm}$ wide, $20 \mathrm{~cm}$ deep hole in the center of the failed digester, 132 and then inoculum was injected into the hole (injection). The inoculum was expected to infiltrate 133 into the surrounding annular space and bottom area. (3) The feedstock was removed and 134 completely mixed with the added inoculum, and then re-loaded into the digester (well-mixing).

135 These three methods were used to mimic possible recovery practices for large-scale digesters.

136 For each digester, a 10-L Tedlar gas bag (CEL Scientific, Santa FeSprings, CA, USA) was 137 attached to collect biogas every 2-5 days during the 5-month experimental period. 
139 Besides the digesters used for the recovery tests, several other digesters were used as the controls

140 (Table 2). Two 1-L digesters (control 1) were operated at a low F/I ratio of 0.5 and a low total

141 solids content of $9.5 \%$, and were on a shaker during the entire digestion process, which was

142 expected to generate close to maximum biogas yield. Two 1-L digesters (control 2) were

143 operated at an F/I ratio of 4 and total solids content of 20; and one 9-L digester (control 3) was

144 operated at an F/I ratio of 10 and was not recovered.

$146 \quad 2.3$ Analytical methods

147 The volume of biogas was measured with a drum-type gas meter (Ritter, Bochum, Germany) and 148 its composition $\left(\mathrm{CH}_{4}, \mathrm{CO}_{2}, \mathrm{~N}_{2}\right.$, and $\left.\mathrm{O}_{2}\right)$ was analyzed with a gas chromatograph (Agilent, $\mathrm{HP}$ 149 6890, Wilmington, DE, USA) equipped with a $30 \mathrm{~m} \times 0.53 \mathrm{~mm} \times 10 \mu \mathrm{m}$ Rt巴 - Alumina $150 \mathrm{Bond} / \mathrm{KCl}$ deactivation column and a thermal conductivity detector. Helium gas was used as a 151 carrier gas at a flow rate of $5.2 \mathrm{~mL} / \mathrm{min}$. The temperature of the detector was maintained at $200^{\circ} \mathrm{C}$, 152 while the initial temperature of the oven was $40^{\circ} \mathrm{C}$ and then increased to $60^{\circ} \mathrm{C}$ within one minute.

153 The TS, VS, pH, and alkalinity of the feedstock and inoculum were measured based on the

154 Standard Methods Examination of Water and Wastewater (APHA, 2005). Specifically, a $5 \mathrm{~g}$ 155 sample was diluted with $50 \mathrm{ml}$ of deionized (DI) water, and then the $\mathrm{pH}$ and alkalinity were 156 measured using an auto-titrating pH meter (Mettler Toledo, DL22 Food \& Beverage Analyzer, 157 Columbus, $\mathrm{OH}, \mathrm{USA})$. Total carbon and total nitrogen contents were determined using an 158 elemental analyzer (Elementar Vario Max CNS, Elementar Americas, Mt. Laurel, NJ, USA). 159 Volatile fatty acids (VFAs), which include propionic, acetic, isovaleric, butyric, isobutyric, and 160 valeric acids, were analyzed using a gas chromatograph (Shimadzu, 2010PLUS, Columbia, MD, 161 USA) equipped with a $30 \mathrm{~m} \times 0.32 \mathrm{~mm} \times 0.5 \mu \mathrm{m}$ Stabilwax ${ }^{\circledR}$ polar phase column and a flame 
162 ionization detector according to a method described previously (Shi et al., 2013). Monomeric

163 sugars (glucose, xylose, galactorse, arabinose, and mannose) were analyzed using high-

164 performance liquid chromatography (Shimadzu, LC-20AB, Columbia, MD, USA) equipped with

165 a Biorad Aminex HPX-87P column and a refractive index detector. HPLC grade water was used

166 as the mobile phase at a flow rate of $0.3 \mathrm{~mL} / \mathrm{min}$, and temperatures of the column and detector

167 were maintained at $60^{\circ} \mathrm{C}$ and $55^{\circ} \mathrm{C}$, respectively. Statistical analysis of the data was conducted

168 using SigmaPlot and R Studio software. Averages and standard errors are reported.

169

170 3. Results and discussion

$171 \quad 3.1$ Performance of the controls

172 As shown in Fig. 2, at a low F/I ratio of 0.5 and low TS of 9.5 with continuous shaking (control

173 1), the daily methane yield peak value (13.3 L/kg-VS.day) was reached on day 13 and methane

174 content stabilized on day 19. The accumulative methane yield was $330.5 \mathrm{~L} / \mathrm{kg}$-VS (Fig. 3). This

175 result is considered as the maximum achievable methane yield, and is close to the results

176 reported by other researchers (Xu et al., 2013). Control 2 and 3 digesters (Table 2), however,

177 quickly failed, producing only 2.4 and $0.4 \mathrm{~L} / \mathrm{kg}-\mathrm{VS}$ of methane, respectively (Fig. 3). Failure at

178 an $\mathrm{F} / \mathrm{I}$ ratio of 10 was expected based on previous studies ( $\mathrm{Xu} \& \mathrm{Li}, 2012$ ); while failure at $\mathrm{F} / \mathrm{I}$

179 ratio of 4 reflects the instability of SS-AD as discussed in the introduction.

180

1813.2 Recovery performance

182 For all treatments, biogas yields were lower than $0.1 \mathrm{~L} / \mathrm{kg}-\mathrm{VS}$ during the first 20 days and,

183 therefore, were considered as failed. The recovery step was carried out on day 20 and 
184 significantly increased methane yield thereafter (Fig. 4). Different patterns in methane yield and 185 methane content were observed for the three recovery methods.

187 With the top-addition method, daily methane yield reached the stable phase 10 days after 188 recovery and lasted for 1 month, and then gradually decreased (Fig. 4A). The peak daily methane 189 yield (1.01 L/kg-VS.day) was much lower than that obtained from recovered digesters using 190 other methods (Fig. 4B and 4C), and also much lower than the control 1 digesters. Based on 191 methane content, the digesters also took a longer time ( 25 days) to reach the stable phase (Fig. 192 4D). The low daily methane yield and long time for stabilization could have been caused by slow 193 infiltration of inoculum. The inoculum was sticky and had a moderate solid content of $8.06 \%$

194 (Table 1), and thus had limited mobility. Even through it was hard to confirm visually, it is likely 195 that the added inoculum did not reach the bottom of digesters by the end of the recovery test, as 196 evidenced by differences in color and moisture content. The bottom layer of the feedstock had a 197 lighter color and lower moisture content compared to the top layer where the inoculum was 198 added. Slow infiltration of inoculum would have reduced access to feedstock for methane 199 production. In addition, aerobic microbes may have been enabled, resulting in the production of 200 other gases, which contributed to the low methane content and yield at the beginning of recovery.

202 With the injection method, the daily methane yield reached the first peak 10 days after recovery 203 was initiated, and then obtained a second peak 33 days after the first peak (Fig. 4B). Compared 204 to the top-addition method, the daily methane yield with injection recovery was about two times 205 higher, which may be because of the larger contact surface area between the feedstock and added 206 inoculum, as the inoculum was introduced to the surrounding annular space and bottom area (Fig. 
1). Possibly, the first peak was generated from the immediately available feedstock in the central

208 and the bottom area where the inoculum was injected; while the second peak may have been

209 caused by the slow infiltration of inoculum. The methane content in biogas slowly increased to

$21055 \% 32$ days after recovery and then remained constant (Fig. 4E). The slow increase of methane

211 content was similar to that observed from the digesters that used the top-addition method, and

212 was very likely caused by the slow infiltration of inoculum. Both methane yield and methane

213 content were highly dynamic as indicated by the large standard errors (Fig. 4B and 4E). These

214 large errors could have been caused by the uneven diffusion and distribution of inoculum in the

215 digesters.

217 With the well-mixing method, the daily methane yield slowly increased to $2.49 \mathrm{~L} / \mathrm{kg}$-VS 54 days

218 after recovery was initiated (Fig. 4C). The peak value was 50\% higher than that obtained from

219 the digesters using the injection method, and 150\% higher than those using the top-addition

220 method. However, it took a much longer time than the other two methods to reach peak yields,

221 and dropped quickly after that. The overall profile of daily methane yield using the well-mixing

222 method was similar to the control 1 digesters (Fig. 2), but with a longer progressing time and a

223 lower yield. The methane content quickly jumped to 53\% in 16 days after recovery, which was

224 shorter than the other two recovery methods, but again similar to the control 1 digesters.

225 Interesting differences were observed when comparing the digesters using the well-mixed

226 method to control 2 digesters. Control 2 digesters, which were well-mixed initially, had a

227 constant F/I ratio of 4 (Table 2), and failed quickly. While the digesters using the well-mixing

228 recovery method, which had an initial F/I ratio of 10 that was decreased to 4, succeeded in

229 producing biogas. The failure of control 2 digesters likely was caused by the over accumulation 
230 of digestion intermediates, e.g. volatile fatty acids and free ammonia, which inhibited

231 methanogens (Yang et al., 2014). While for the recovery test, the inoculum added initially (F/I

232 ratio of 10), although not able to produce biogas, should have created a suitable digestion

233 environment in which volatile fatty acids were partially consumed by aerobic or anaerobic

234 microbes, increasing the overall alkalinity. Therefore, when inoculum was added for recovery,

235 inhibition on methanogens may not have been as extensive as in the control 2 digesters, so that

236 methanogens grew slowly as indicated by the long lag phase as shown in Fig. 4C.

238 The cumulative methane yields from all controls and treatments are shown in Fig. 3. The

239 methane yields from controls 2 and 3 were negligible. The Student's t-test results showed that all

240 three recovery methods significantly improved methane yield compared to the control 3 digesters

$241 \quad(p<0.01)$. Based on this test only, the well-mixing method recovered the highest amount of

242 methane, followed by the injection and top-addition methods. The methane yields from the

243 recovered digesters were equal to $30-40 \%$ of that produced from control 1 digesters, which had a

244 low F/I ratio of 0.5 and a low TS content of 9.5 and were shaken continuously. If more inoculum

245 was used for recovery, it is highly likely that more methane could be produced.

$247 \quad 3.3$ Limiting factors of recovery

248 Based on the pattern of methane production after recovery, the slow infiltration of inoculum

249 seemed to be a major obstacle for the top-addition and injection methods. In another words, the

250 limiting factor was the slow mass transfer. Methods such as multiple layer addition and multiple

251 injection holes would increase contact between inoculum and feedstock, and, therefore, may

252 improve methane yield. For the well-mixing recovery method, the contact between inoculum and 
253 feedstock was not a problem, but the presence of inhibitory agents and the slow growth of

254 digestion microbes were. One possible way to solve these problems is to increase the amount of 255 inoculum for recovery, which would dilute inhibitors and provide sufficient digestion microbes.

257 Besides methane yield, selection of a recovery method would also be affected by other factors, 258 such as energy, space outside the digester, and equipment requirements as well as available space 259 inside the digester. Top-addition and injection methods, even with multiple layers or holes, are 260 relatively simple; whereas, the well-mixing method is energy consuming and usually needs extra 261 space as it can be difficult to mix inoculum and feedstock inside of a failed digester due to the 262 high solids content. All three recovery methods are affected by the availability of space in the 263 digester. In this test, the amount of inoculum added for recovery (to decrease F/I ratio 4) was 264 marginal but was still able to resume biogas production. Therefore, it is envisaged that recovery 265 of failed digesters can be feasible for large scale solid-state anaerobic digesters.

\section{4. Conclusion}

268 All three recovery methods resulted in resumed methane production at an F/I ratio of 4 . Overall 269 methane yields from recovered digesters were equal to $30-40 \%$ of that obtained from the control 270 digesters with a low F/I ratio of 0.5 , and outperformed control digesters that had a constant F/I 271 ratio of 4 . The well-mixing method showed the highest methane yield, followed by injection and 272 top-addition methods. Slow mass transfer could be the limiting factor for top-addition and 273 injection methods, while slow growth of digestion microbes may have obstructed well-mixed 274 digesters. 


\section{Acknowledgement}

276 This project was funded by USDA NIFA Biomass Research and Development Initiative Program

277 (Award No. 2012-10008-20302). The authors wish to thank Mrs. Mary Wicks (Department of

278 Food, Agricultural and Biological Engineering, OSU) for critical review.

279

280 References

281 Aboudi, K., Álvarez-Gallego, C.J., Romero-García, L.I.R. 2016. Biomethanization of sugar beet byproduct by semi-continuous single digestion and co-digestion with cow manure. Biores. Tech., 200, 311-319.

APHA. 2005. Standard Methods for the Examination of Water and Wastewater. 21st Ed., American Public Health Association. Washington, D.C.

Bollon, J., Le-Hyaric, R., Benbelkacem, H., Buffiere, P. 2011. Development of a kinetic model for anaerobic dry digestion processes: Focus on acetate degradation and moisture content. Biochem. Eng. J., 56(3), 212-218.

Fagbohungbe, M.O., Dodda, I.C., Herbertb, B.M.J., Li, H., Ricketts, L., Semple, K.T. 2015. High solid anaerobic digestion: Operational challenges and possibilities. Environ. Technol. Innov., 4, 268-284.

292 Graef, S.P., Andrews, J.F. 1974. Stability and control of anaerobic digestion. Water Pollut. Control Federat., 46(4), 666-683.

294 Kafle, G.K., Kim, S.H. 2011. Sludge exchange process on two serial CSTRs anaerobic 295 digestions: Process failure and recovery. Biores. Tech., 102(13), 6815-6822. 
Karagiannidis, A., Perkoulidis, G. 2009. A multi-criteria ranking of different technologies for the anaerobic digestion for energy recovery of the organic fraction of municipal solid wastes. Biores. Technol., 100(8), 2355-2360.

Karthikeyan, O.P., Visvanathan, C. 2013. Bio-energy recovery from high-solid organic substrates by dry anaerobic bio-conversion processes: A review. Rev. Environ. Sci. Biotechnol., 12(3), 257-284.

Li, L., He, Q., Wei, Y., He, Q., Peng, X. 2014. Early warning indicators for monitoring the process failure of anaerobic digestion system of food waste. Biores. Tech., 171, 491-494.

Li, Y., Zhu, J., Wan, C., Park, S.Y. 2011. Solid-state anaerobic digestion of corn stover for biogas production. Trans. ASABE, 54(4), 1415-1421.

Liew, L.N., Shi, J., Li, Y. 2011. Enhancing the solid-state anaerobic digestion of fallen leaves through simultaneous alkaline treatment. Biores. Tech., 102(19), 8828-8834.

Sheets, J.P., Yang, L., Ge, X., Wang, Z., Li, Y. 2015. Beyond land application: Emerging technologies for the treatment and reuse of anaerobically digested agricultural and food waste. Waste Manag., 44, 94-115.

Shi, J., Wang, Z.J., Stiverson, J.A., Yu, Z.T., Li, Y.B. 2013. Reactor performance and microbial community dynamics during solid-state anaerobic digestion of corn stover at mesophilic and thermophilic conditions. Biores. Tech., 136, 574-581.

Vavilin, V.A., Fernandez, B., Palatsi, J., Flotats, X. 2008. Hydrolysis kinetics in anaerobic degradation of particulate organic material: An overview. Waste Manag., 28(6), 941-953.

Verma, S. 2002. Anaerobic digestion of biodegradable organics in municipal solid wastes. . in: Department of Earth and Environmental Engineering, Fu Foundation School of Engineering and Applied Science, Columbia University. 
319 Xu, F., Li, Y. 2012. Solid-state co-digestion of expired dog food and corn stover for methane $320 \quad$ production. Biores. Technol., 118, 219-226.

321 Xu, F.Q., Shi, J., Lv, W., Yu, Z.T., Li, Y.B. 2013. Comparison of different liquid anaerobic 322 digestion effluents as inocula and nitrogen sources for solid-state batch anaerobic digestion of corn stover. Waste Manag., 33(1), 26-32.

324 Yang, L., Xu, F., Ge, X., Li., Y. 2014. Challenges and strategies for solid-state anaerobic 325 digestion of corn stover. Biores. Tech., 175, 430-435. 
7

\begin{tabular}{|c|c|c|}
\hline Parameters & Feedstock & Inoculum \\
\hline $\mathrm{TS}, \%$ & $89.14 \pm 0.43$ & $8.06 \pm 0.01$ \\
\hline VS, \% & $84.05 \pm 0.61$ & $3.94 \pm 0.01$ \\
\hline $\mathrm{TC}, \%$ & $43.40 \pm 0.23$ & $2.60 \pm 0.07$ \\
\hline $\mathrm{TN}, \%$ & $0.60 \pm 0.00$ & $0.32 \pm 0.01$ \\
\hline $\mathrm{C} / \mathrm{N}$ ratio & $71.86 \pm 0.75$ & $8.19 \pm 0.59$ \\
\hline $\mathrm{pH}$ & $6.41 \pm 0.02$ & $8.00 \pm 0.01$ \\
\hline Alkalinity, g- $\mathrm{CaCO}_{3} / \mathrm{kg}$ & $0.19 \pm 0.00$ & $10.76 \pm 0.21$ \\
\hline VFAs, g/kg & $0.27 \pm 0.02$ & $0.93 \pm 0.06$ \\
\hline Cellulose $^{\text {a }}, \%$ & $34.40 \pm 0.35$ & $1.75 \pm 0.13$ \\
\hline Hemicellulose ${ }^{a}, \%$ & $20.61 \pm 0.05$ & $1.01 \pm 0.00$ \\
\hline
\end{tabular}


Table 2 Operating conditions of digesters

\begin{tabular}{cccccccc}
\hline \multirow{2}{*}{ Conditions } & \# of digesters & $\begin{array}{c}\text { Digester Vol } \\
\text { (L) }\end{array}$ & \multicolumn{2}{c}{ Initial condition } & \multicolumn{2}{c}{ After recovery } & $\begin{array}{c}\text { Operation time } \\
\text { (months) }\end{array}$ \\
\cline { 4 - 7 } & & 9 & F/I & TS & F/I & TS & 5 \\
\hline Top-addition & 2 & 9 & 10 & 32 & 4 & 20 & 5 \\
Injection & 2 & 9 & 10 & 32 & 4 & 20 & 5 \\
Well-mixing & 2 & 1 & 0.5 & 9.5 & No recovery & 2 \\
\hline Control 1 & 2 & 1 & 4 & 20 & No recovery & 2 \\
Control 2 & 2 & 9 & 10 & 32 & No recovery & 5 \\
Control 3 & 1 & & & & & & 5 \\
\hline
\end{tabular}

353

354

355

356

357

358 

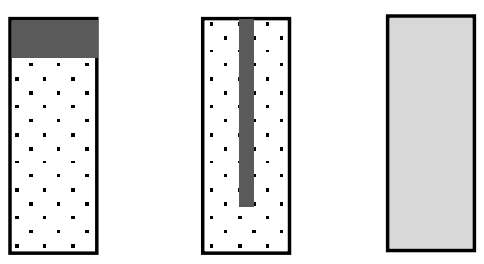

(a)

(b)

(c)

363 Fig. 1 SS-AD digester recovery methods. a): top-addition, b): injection, and c): well-mixing.

364

365

366

367

368

369

370

371

372

373

374

375 


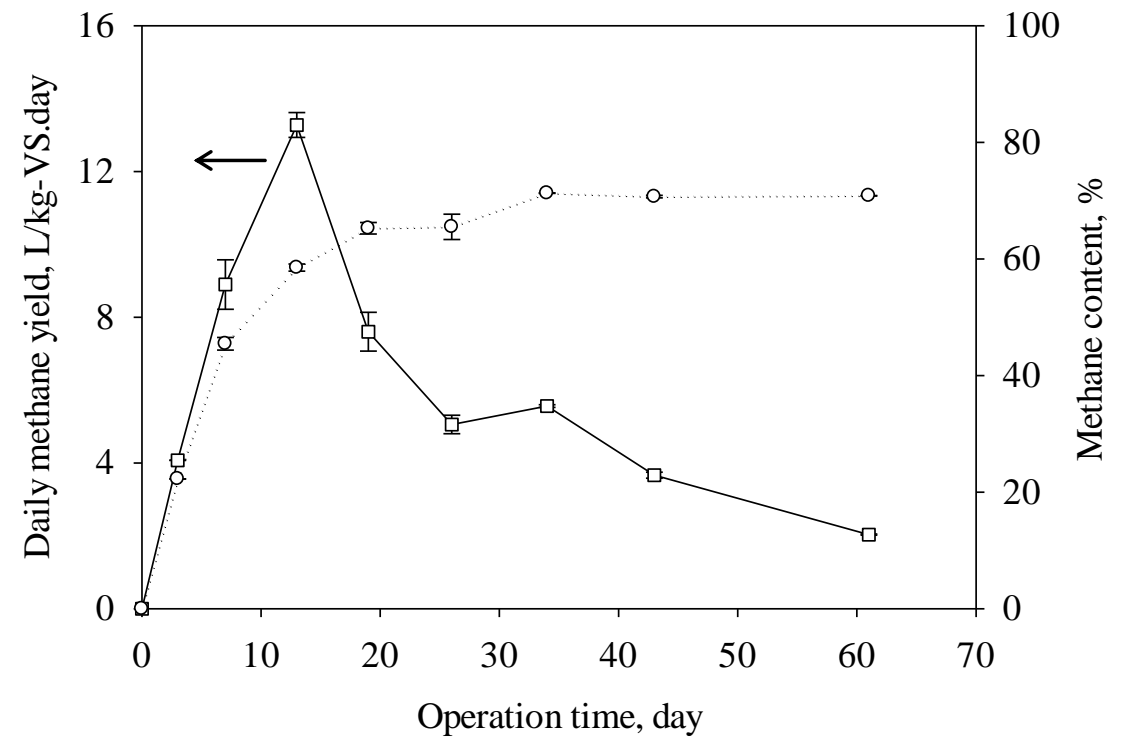

377 Fig. 2 Daily methane yield and methane content of digesters with $\mathrm{F} / \mathrm{I}=0.5$ (control 1 digesters). 


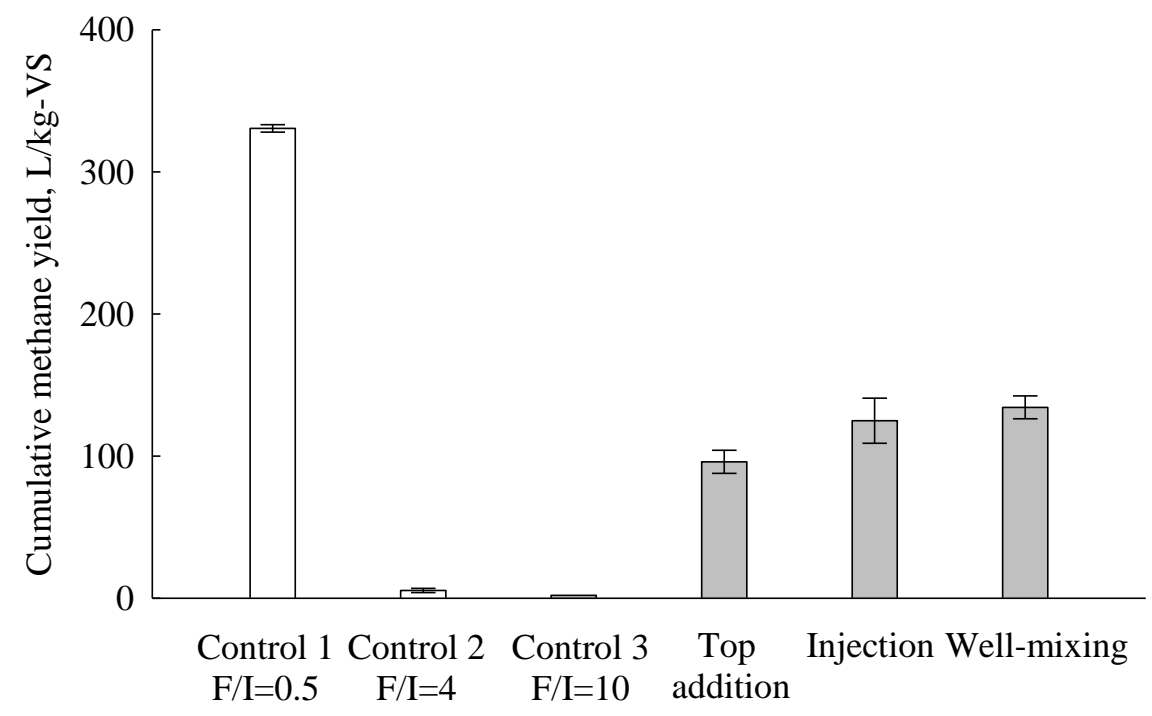

394 Fig. 3 Comparison of cumulative methane yield from the control and recovered digesters.

395

396

397 

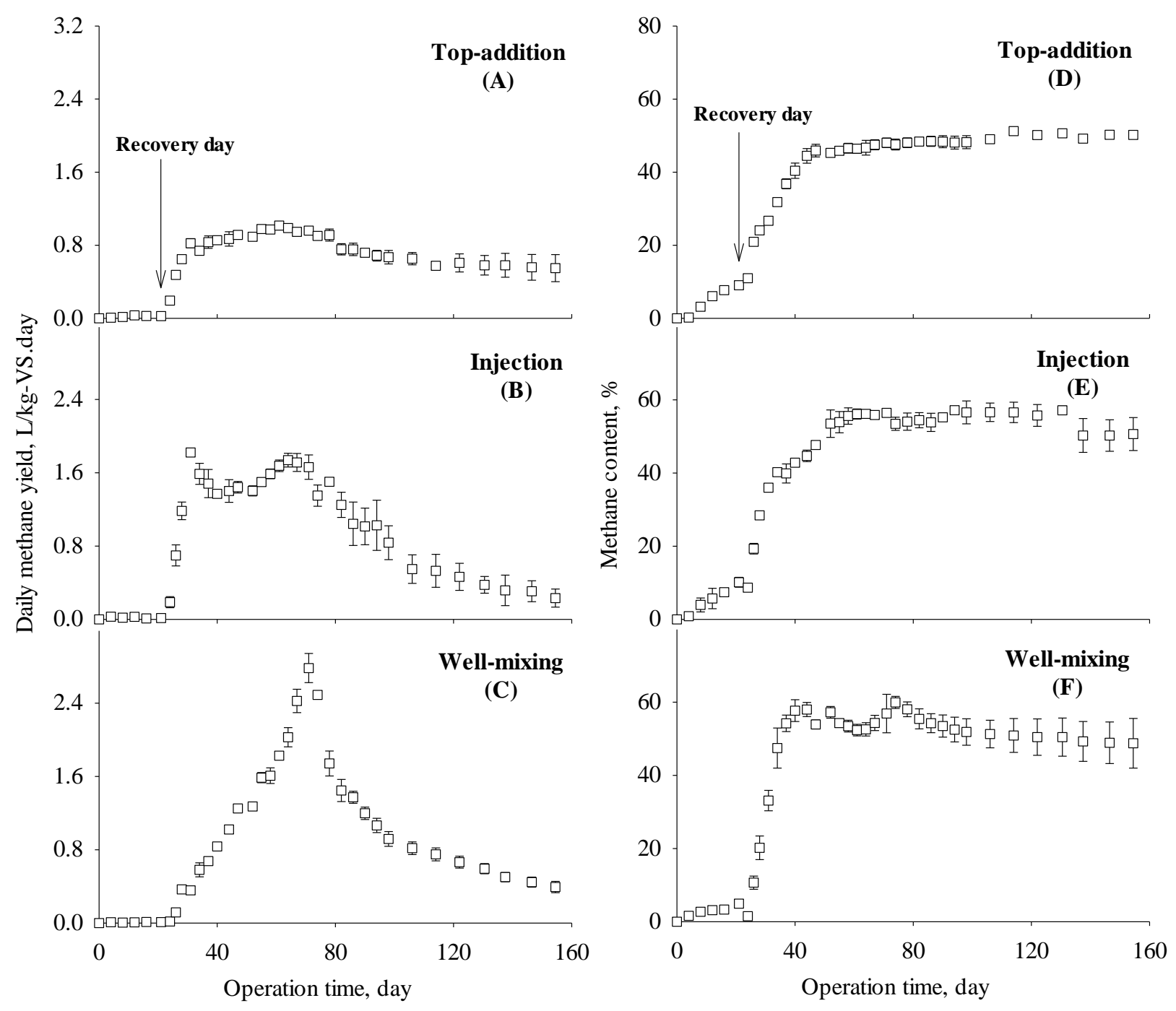

399 Fig. 4 Daily methane yield (A, B, C) and methane content (D, E, F) from recovered digesters. 400 The recovery process was initiated on day 20. 